\title{
ON THE SCALING OF SMALL, HEAT SIMULATED JET NOISE MEASUREMENTS TO MODERATE SIZE EXHAUST JETS
}

\author{
Dennis K. McLaughlin, ${ }^{1}$ \\ Penn State University, University Park, PA 16802 \\ James Bridges, ${ }^{2}$ \\ NASA Glenn Research Center, Cleveland, OH 44135 \\ Ching-Wen $\mathrm{Kuo}^{3}$ \\ Penn State University, University Park, PA 16802
}

\begin{abstract}
Modern military aircraft jet engines are designed with variable geometry nozzles to provide optimum thrust in different operating conditions, depending on the flight envelope. However, the acoustic measurements for such nozzles are scarce, due to the cost involved in making full scale measurements and the lack of details about the exact geometry of these nozzles. Thus the present effort at The Pennsylvania State University and the NASA Glenn Research Center in partnership with GE Aviation is aiming to study and characterize the acoustic field produced by supersonic jets issuing from converging-diverging military style nozzles. An equally important objective is to validate methodology for using data obtained from small and moderate scale experiments to reliably predict the most important components of full scale engine noise. The experimental results presented show reasonable agreement between small scale and moderate scale jet acoustic data, as well as between heated jets and heat-simulated ones. Unresolved issues however are identified that are currently receiving our attention, in particular the effect of the small bypass ratio airflow. Future activities will identify and test promising noise reduction techniques in an effort to predict how well such concepts will work with full scale engines in flight conditions.
\end{abstract}

\section{Nomenclature}

$a \quad=$ ambient speed of sound

$A R \quad=$ exit-to-throat area ratio

$D \quad=$ diameter of nozzle at exit plane

$D_{j} \quad=$ diameter of the jet plume

$f \quad=$ frequency

$f_{c} \quad=$ characteristic frequency $U_{j} / D_{j}$

$M_{a} \quad=$ acoustic Mach number $U_{j} / a_{\infty}$

$M_{d} \quad=$ design Mach number of the nozzle

$M_{j} \quad=$ average Mach number of the fully expanded jet

$N P R=$ nozzle pressure ratio

$O A S P L=$ overall sound pressure level

$R \quad=$ physical distance of the microphones from the jet exit

$\Re=$ gas constant

$R_{e} \quad=$ Reynolds number

\footnotetext{
${ }^{1}$ Professor, Department of Aerospace Engineering, AIAA Fellow.

${ }^{2}$ Senior Research Scientist, NASA Glenn Research Center, Senior Member AIAA.

${ }^{3}$ Graduate Research Assistant, Department of Aerospace Engineering, AIAA Student Member.
} 


$\begin{array}{ll}\mathfrak{R}_{\infty} & =\text { ambient gas constant } \\ P S D & =\text { power spectral density } \\ T_{\infty} & =\text { ambient temperature } \\ T_{o} & =\text { jet stagnation temperature } \\ T T R & =\text { total temperature ratio, } T_{o} / T_{\infty} \\ S t & =\text { Strouhal number } \\ U_{j} & =\text { mean exhaust velocity } \\ x & =\text { physical downstream distance from the nozzle exit plane } \\ \text { y } & =\text { physical parallel distance from the jet centerline } \\ \Delta f & =\text { frequency resolution bandwidth } \\ \rho & =\text { density } \\ \theta & =\text { polar angle, measured from the jet downstream direction }\end{array}$

Subscripts:

heated : quantity relative to the heated jet

$j \quad$ : quantity relative to the jet

raw : as measured

\section{Introduction}

As the engine power of airplanes rises and the frequency of aircraft operations increases, environmental laws are becoming more stringent. While jet noise is not the dominant contributor to the total civilian aircraft noise it once was, it is still a major contributor. Jet noise is the major noise source for military aircraft that have engines of very low bypass ratio and high exit flow temperatures and velocities. The resulting noise poses a health threat to ground crews as well as causes an annoyance to communities in the vicinity of military airbases. Noise control and monitoring for both civil and military airplanes are also growing. Because practical aircraft engine measurements are very time consuming and subject to large uncertainties, there is strong interest in developing more accurate small or moderate model scale experiments which can be conducted in more controlled environments. Clearly it is much cheaper to develop jet noise reduction technologies on small-scale rigs than full-scale engines. However, experimental consistency between model scale and actual engine radiated noise measurements has yet to be fully demonstrated.

This study is part of an effort led by The Pennsylvania State University and the NASA Glenn Research Center (GRC) with partner GE Aviation. The aim of this study was to validate the methodology for using data obtained from testing at small and moderate scale, supported by computations, to reliably predict the full scale engine noise. The approach was to conduct and compare small scale and moderate scale experiments with nozzles representative of military jet engine exhaust nozzles. Comparisons across scales with data obtained in different facilities will provide confidence in the quality of the measurements performed, and in the ability of the methodology to extrapolate the subscale data to the full size aircraft. This paper focuses on making extensive comparisons between small scale measurements performed at Penn State and moderate scale data gathered at NASA GRC, in order to determine whether the small scale and heat simulated jets can accurately simulate the acoustics issuing from moderate scale hot jets. A similar study was conducted by Viswanathan [1] that presented the results of numerous comparisons of moderate size (predominantly) subsonic jets' acoustic measurements. Additionally this work showed comparisons of model supersonic jet acoustic experiments with those of a turbojet engine with an acoustically treated duct between the turbomachinery and the exhaust nozzle. These comparisons showed favorable agreement for both subsonic and supersonic jet flows.

The experiments were performed in two laboratories: the Jet Noise Laboratory at Penn State University and the AeroAcoustic Propulsion Laboratory at NASA GRC. The High Flow Jet Exit Rig (HFJER) at NASA GRC is one of the largest such facilities in the country. Supersonic air jets with exit diameters of approximately $10 \mathrm{~cm}$ are commonly used with jet Mach numbers up to $M_{j}=1.7$ and stagnation temperatures exceeding three times standard ambient temperature. The NASA GRC large anechoic geodesic hemispherical dome has a radius of approximately 20 meters. The small scale experiments were performed in the Jet Noise Laboratory at Penn State, with its university size anechoic chamber (with dimensions of $5 \times 6 \times 2.8$ meters). Typical supersonic jet nozzles of exit diameters from 2 to $2.5 \mathrm{~cm}$ that operate at pressure ratios up to 4.5 producing exit Mach numbers up to $M=1.7$. This facility does not include jet heating capability. Instead helium-air mixture jets are used to simulate hot jets. Appropriate mixtures of helium-air can be made to have the desired acoustic velocity of heated jets. When operated at the same 
pressure ratio of heated air jets, the elevated acoustic velocities also have proportionally increased jet velocities. It is this property that the radiated noise best correlates with. Doty and McLaughlin [2] and Papamoschou [3] have shown that mixtures of helium and air can be used to simulate the flow and acoustic properties of hot jets with a very high degree of accuracy.

\section{Experimental Facilities, Set-Up, and Procedure}

\section{A. Penn State facility and instrumentation}

The Pennsylvania State University high speed small scale jet noise facility was used for the small scale experiments presented in the current study. The facility schematic is shown in Fig. la. High pressure air, pressurized by a $C S$ 121 compressor combined with a KAD-370 air dryer both manufactured by Kaeser Compressors, is provided from the tank, and then the air flow is regulated via pressure regulators and control valves located in a piping cabinet before being fed to a plenum and delivered to the jet nozzle issuing into the anechoic chamber. A pitot probe is embedded in the middle section of the plenum to provide, via a pressure transducer, the total pressure upstream of the nozzle. The helium supply piping is connected to the piping cabinet to provide the helium-air mixture jets in order to simulate the heated jets. The individually partial pressures of the helium and air are both regulated in the piping cabinet. This operating procedure was demonstrated and developed by Doty and McLaughlin [2] over 10 years ago. The anechoic chamber walls are covered with fiberglass wedges and it has an approximate cut-off frequency of $250 \mathrm{~Hz}$. An exhaust collector and fan on the opposite wall of the plenum in the anechoic chamber prevents flow circulation and possible helium accumulation.

Acoustic measurements are currently performed using six microphones, hanging from a boom that extends from the plenum stand, as can be seen in the image of Fig. lb. The microphone array is set and aimed to freely rotate around a point located at the center of the nozzle exit plane. The microphones are positioned at a grazing incidence to the jet centerline and equally spaced by 10 degrees. The averaged physical radial distance of microphones to the nozzle exit is around $1.78 \mathrm{~m}$ (70 in). This distance is appropriate enough for the microphones to be considered in the far field when testing nozzles less than $2.5 \mathrm{~cm}(1 \mathrm{in})$ in diameter operated in this facility. The microphones are $1 / 8$ " pressure-field microphones selected to match the testing nozzles operated at the small scale facility, type 4138 from Brïel and Kjaer ( $B \& K$ ), and type 40DP from GRAS. Following calibration corrections the acoustic data have a frequency response reliably accurate to $120 \mathrm{kHz}$. This is adequate to define the acoustic frequencies most important to noise studies including those approximately a factor of 10 higher than the peak frequencies in the maximum noise emission direction. The range is not adequate to fully define the noise spectra to the highest non-dimensional frequencies that are typically much less important in supersonic aircraft noise. The acoustic measurements were performed from polar angle $\theta=30^{\circ}$ to $\theta=120^{\circ}$ measured from the jet downstream direction originated from the nozzle exit plane, with increments of 10 degrees. The schematic of the Penn State data acquisition process is shown in Fig. 2. The microphone calibration is performed with a $B \& K$ acoustic calibrator, model 4231, and the microphone calibration constants are recorded to provide the conversion from the measured voltages to the equivalent pressure. The analog time-domain signals from the microphones are routed through a Nexus, $B \& K$ signal conditioner or a GRAS model $12 \mathrm{AN}$ power module and then amplified and filtered for antialiasing thus enabling their accurate digital conversion in the following acquisition. A high-pass filter is also set to $500 \mathrm{~Hz}$, removing any undesirable low frequency noise that could contaminate the data. A PCI-6123 National Instruments DAQ board is installed in the operating computer acquiring the time domain data stored in binary files. The data acquisition and storing is accomplished with LabVIEW software. The sampling rate is set at $300 \mathrm{kHz}$ for the data acquisition and 102,400 to 409,600 data point are collected, the reduced data set being used for helium-air mixture jets in order to reduce the amount of helium used during an experiment. The raw data are then fed into Matlab for the data processing. The raw data are sequentially split into 1024 or 4096 points segments and a Hanning window function is applied with 50 percent overlap between each window. The Fast Fourier Transform (FFT) is calculated in each window and the value is averaged from the 199 segments. This yields the power spectral density $(P S D)$ which is then converted to decibels (dB) using a reference pressure of $20 \mu \mathrm{Pa}$.

\section{B. NASA GRC facility and instrumentation}

Comparisons are presented in this study with acoustic measurements from similar nozzles at a larger scale acquired at the NASA GRC AeroAcoustic Propulsion Laboratory with the high flow jet exit rig (HFJER) as shown in Fig. 3. This facility has a hot jet acoustic rig located in the anechoic geodesic hemispherical dome noted earlier. Jet diameters up to $12.7 \mathrm{~cm}(5 \mathrm{in})$ are typically used in this facility for supersonic applications, with the capability to use 
a co-flow to simulate jets with a small bypass ratio according to the design evolution of modern military engine. The facility can be operated in a single-flow mode as well as a dual-flow mode. When in the dual-flow mode the pressure in both the annular and the core section of the plenum are set to the same value; however, the annular flow was unheated when performing hot jet measurements. A schematic diagram of this dual flow set-up is shown in Fig. 4. For the experiments reported in this paper, the cold bypass air comprised $30 \%$ of the core flow, a value that is roughly what is used in turbojet engines on today's military fighter aircraft. The total temperature ratio of the jets is expressed in terms of the core flow total temperature $T T R_{\text {core }}$, or the total temperature of the flow after it is (hypothetically) fully mixed, $T T R_{m i x}$. Extensive description and qualification measurements of this facility can be found in Bridges and Brown [4]. Because the pressure upstream of the nozzle throat in the bypass stream is the same as in the core flow, a reasonable assumption is that the exit Mach numbers of both streams is the same, while the TTR of the annular stream is $\sim 1$. An arc of microphones with radius approximately $15 \mathrm{~m}(49 \mathrm{ft})$ is mounted inside the top of the dome with 24 microphones separated by 5 degree increments.

In addition to the dual flow jet facility, a small hot jet acoustic rig (SHJAR) [5] also located in the AeroAcoustic Propulsion Laboratory, provided additional single stream hot jet measurements for the current studies. This rig utilizes a microphone arc with 24 microphones separated by 5 degree increments at a radius of $2.5 \mathrm{~m}$ (100 in). The nozzle exit diameter used for this rig is $5.08 \mathrm{~cm}(2 \mathrm{in})$, thus $R / D=50$ for these experiments.

In both NASA rigs the microphones were Bruel and Kjaer 1/4" microphones (model 4939 with a $B \& K$ Model 2670 preamp), used without grid caps for maximally flat response and pointed at the nozzle exit. Bruel \& Kjaer Nexus ${ }^{\mathrm{TM}}$ amplifiers provided the signal conditioning. A DataMAX ${ }^{\circledR}$ Instrumentation Recorder, from RC Electronics, simultaneously recorded data from all microphones, using a $90 \mathrm{kHz}$ low-pass filter to limit the bandwidth (at $200 \mathrm{kHz}$ sample rate). Eight seconds of data were recorded at each point. Jet flow conditions were recorded during the acoustic data acquisition using a facility computer. Variables such as rig temperatures, pressures, and mass flows, as well as ambient temperature, pressure, and humidity were averaged over the same time that the acoustic record was recorded. The facility data were imported, along with the raw acoustic time series files, into a Linux processing workstation for processing. The processing steps mirror those of PSU facility. Recorded time series were multiplied by amplification factors from the variable gain Nexus signal conditioners and by the calibration factor determined by in situ recording of the Bruel and Kjaer model 4220 pistonphone. Fourier transforms were computed using FFT algorithms and $10^{\text {th }}$ order Kaiser-Bessel windowing on 16384-point records with $50 \%$ overlapping. Power spectral density estimates were created by averaging all 195 transforms and adding back windowing losses, and the resulting PSD were converted to decibels relative to $20 \mu \mathrm{Pa}$. Background noise, measured immediately before the data set was acquired, was subtracted and any frequency band within $3 \mathrm{~dB}$ of the background was flagged and was not considered in future processing and final plotting.

\section{Data processing and comparison procedure}

In both PSU and NASA processing systems, three corrections are applied to the raw PSD to compute the normalized, lossless $P S D$. Data were corrected for microphone spectral response characteristics based on the manufacturer's documentation of each individual microphone obtained during factory calibration $\left(\Delta C_{a c t}(H z)\right)$ including appropriate free-field response $\left(\Delta C_{f f}(\mathrm{~Hz})\right)$. The spectra were corrected for daily variations in atmospheric attenuation by calculating the attenuation (ISO 9613-2:1996) for each microphone using measured ambient pressures, humidities, and temperatures $\left(\Delta C_{a t m}(H z)\right)$ and adding back the sound lost due to atmospheric attenuation from the jet to the microphone. Finally, the spectra are non-dimensionalized to $P S D$ per Strouhal number $\left(10 \times \log _{10} f_{C}\right)$. The Strouhal number is defined as $S t=f / f_{c}$, with $f_{c}$ the characteristic frequency of the jet defined by $f_{c}=U_{j} / D_{j}$, where $\boldsymbol{U}_{j}$ is the jet velocity, and $D_{j}$ is the fully expanded diameter of the jet. Eq. (1) summarizes the different steps that lead to the PSD per unit Strouhal number as explained in Kuo, Veltin and McLaughlin [6].

$$
\underbrace{P S D(S t)}_{\text {Lossless Spectrum }}=\underbrace{P S D_{\text {raw }}(H z)}_{\text {Raw Spectrum }}-\underbrace{\Delta C_{\text {act }}(H z)-\Delta C_{f f}(H z)}_{\text {Microphone Respones }}+\underbrace{\Delta C_{\text {atm }}(H z)}_{\begin{array}{c}
\text { Atmospheric } \\
\text { Correction }
\end{array}}+\underbrace{10 \log _{10} f_{C}}_{\begin{array}{c}
\text { Strouhal Number } \\
\text { Scaling }
\end{array}}
$$

From the $P S D$, given at intervals of $\triangle f$, the $O A S P L$ is calculated via the following equation:

$$
O A S P L=10 \log _{10}\left[\Delta H z \sum 10^{\left(\frac{P S D(H z)}{10}\right)}\right]=10 \log _{10}\left[\Delta S t \sum 10^{\left(\frac{P S D(S t)}{10}\right)}\right]
$$


The experimental data were processed into lossless spectra per unit Strouhal number to make comparison easier across scales. Most measurements were made at distances close to $R_{\text {raw }}=100 \mathrm{D}$ or $144 \mathrm{D}$ depending on the nozzle diameter. Following processing, the resulting data were (back) propagated to $R=100 D$ assuming spherical spreading of the acoustic field to allow direct comparison of data at a common observer distance. This "back" propagated $P S D$ is determined from

$$
P S D(S t)=P S D(S t)+20 \log _{10}\left(R_{\text {raw }} / R\right)
$$

\section{Heated jet simulation}

The density characteristics of heated jets are replicated using gas mixtures in order to produce acoustic measurements in cold small scale facilities that can be directly compared to hot moderate scale experiments or actual aircraft engine measurements. Doty and McLaughlin [2] and Papamoschou [3] have shown that mixtures of helium and air can appropriately simulate the noise of heated jets to a reasonable accuracy by matching density of the heated gas. The features of heated jets are lowered density and increased velocity relative to the speed of sound, and both of these features can be achieved by helium-air mixture jets. However, both parameters, the acoustic velocity $a$ and the density $\rho_{j}$ cannot be precisely matched simultaneously. Thus, two independent methods were developed to match either the density $\rho_{j}$ or the acoustic velocity $a$ from the heated jets. Although there are two matching methods in heated jet simulation, both methods generate very close results. Two matching methods were introduced by Doty and McLaughlin [2] and are briefly described below.

The first methodology consists in matching the acoustic velocity between a helium-air mixture and a corresponding hot air jet.

$$
a_{\text {heated }}=\sqrt{\gamma \Re T_{j}} \leftrightarrow a_{\text {mix }}=\sqrt{\gamma_{\text {mix }} \Re_{\text {mix }} T_{j_{\text {mix }}}}
$$

$\gamma_{\text {mix }}$ and $\mathfrak{R}_{\text {mix }}$ are dependent on the helium concentration. The proper molar mass of helium can easily be calculated in order to equate $a_{\text {heated }}$ and $a_{m i x}$. From there, the partial pressure of helium and air can be computed and the pressure regulators adjusted for the experiment.

The second method consists in matching the density between a heated jet and a helium-air mixture jet, as shown below.

$$
\left(\frac{\rho_{j}}{\rho_{\infty}}\right)_{\text {heated }}=\left(\frac{T_{\infty}}{T_{j}}\right)_{\text {heated }} \leftrightarrow \frac{\rho_{j_{\text {mix }}}}{\rho_{\infty}}=\frac{T_{\infty} \mathfrak{R}_{\infty}}{T_{j_{\text {mix }}} \mathfrak{R}_{\text {mix }}}=\frac{\mathfrak{R}_{\infty}}{\mathfrak{R}_{\text {mix }}}\left[1+\frac{\gamma_{\text {mix }}-1}{2} M^{2}\right]
$$

The molar mass of helium required to equate $\rho_{j \text { mix }}$ with $\rho_{j}$ can be calculated, and from it the partial pressure of helium is once again derived. The two matching methods typically lead to slightly different values for the partial pressures of the mixture. However, as mentioned, experimental results $[2,3]$ showed that the two methods result in acoustic spectra in agreement within $1 \mathrm{~dB}$ across the spectra, and all data presented here uses the acoustic velocity matching method.

\section{E. Model geometry of military style supersonic nozzle}

Besides the experimental results from contoured converging-diverging (CD) nozzle, this study presents the experimental results conducted with the military style nozzles representative of the exhaust of aircraft engines of the F404 family. The exact inner contours of these military style nozzles are the property of General Electric and were provided under an ongoing contract for the Strategic Environmental Research and Development Program (SERDP). While such military engines possess nozzles with variable geometry, adapting to different flight regimes, for this specific research, three nozzles with different exit-to-throat area ratios were used, as specified by GE Aviation. These nozzles were designed with a multi-faceted inside conical contour. At Penn State, the nozzles were fabricated via a rapid prototyping technique (stereo lithography); meanwhile, the nozzles of identical inner geometry but seven times larger, built by GE Aviation, were tested at NASA GRC for the moderate scale experiments. These military style supersonic nozzles (GE nozzles) were built with the identical inner geometry at small and moderate scale to demonstrate the scaling of small heat simulated jets to moderate and full size jets (although no full-scale data are presented in this paper). More details about these military style supersonic nozzles can be found in Kuo et al. [7]. In general, the expansion portion of the flow contour consists of 12 flat segments that are interleaved to facilitate 
area adjustment of the operational nozzles. Unlike well designed contoured CD nozzles, imperfections at the nozzle exit plane contain weak shock cells, even at perfectly balanced pressure conditions.

\section{Experimental Results}

The operating conditions of the data acquired both at Penn State and at NASA GRC are summarized in Table 1. In this table, the jet Mach number $M_{j}$ relates to the average Mach number of the fully expanded jets and the nozzle total temperature ratio $(T T R)$ is the ratio of the jet stagnation temperature $T_{o}$ and the ambient temperature $T_{\infty}$. For the data from NASA GRC, when operating the dual flow rig, the values in the column of TTR are accordingly the values of $T T R_{\text {core }}$ where the subscript core relates to the quantities relative to the core flow and $T T R_{\text {mix }}$ where the subscript mix relates to the quantities relative to the mixture of the annular and core flow. The values of Reynolds number in this table were computed based on the calculation methodology described by Doty and McLaughlin [2]. As a first step, comparisons were made between cold, pure air jet measurements obtained at Penn State with similar measurements from NASA GRC. Then, the heat simulated small scale results are compared to the moderate scale hot jet measurements provided by NASA GRC, with discussions of the results included.

\section{A. Unheated jets measurements}

First, comparisons are made between acoustic spectra from cold jets, between small scale measurements acquired at Penn State and moderate scale measurements from NASA GRC. This allows for a direct cross-scale comparison, from jets issuing from nozzles of different sizes but exactly the same geometry, without adding the complication of the heat simulation. Sample spectra are shown in Figs. 5 and 6 from two testing nozzles: both jets are operated with a pressure ratio $N P R=4$, one of them issuing from the $M_{d}=1.65(A R=1.295) \mathrm{GE}$ nozzle, and the other from the $M_{d}$ $=1.5(A R=1.18) \mathrm{GE}$ nozzle. The convention adopted here is that $M_{d}$ is calculated from the known area ratio assuming the Mach number that would be achieved with ideal quasi parallel flow that has been expanded through the given area ratio. In the figure, the Penn State data were acquired with nozzles $1.8 \mathrm{~cm}(0.7 \mathrm{in})$ in diameter, and the NASA GRC spectra were acquired at with nozzles 7 times larger. The spectra are spaced apart in pairs by $20 \mathrm{~dB}$ for clear elucidation as the polar angle varies and all acoustic levels have been scaled to $R / D=100$ assuming far field spherical spreading. From both figures the cross-scale spectra comparison exhibits very encouraging results, with spectra from the two rigs agreeing within $+/-2 \mathrm{~dB}$ over frequencies $0.05<S t<4$ and angles $30^{\circ}<\theta<120^{\circ}$ as measured from the exhaust axis. The OASPL comparisons consistently present good agreement within $2 \mathrm{~dB}$ variance across the polar angles. It is noted that this agreement is obtained for jet noise spectra containing not only jet mixing noise, but also significant broadband shock noise from jets operating in the over- and under-expanded conditions. This degree of agreement between the data acquired from the two facilities provides a check on the scaling methodology, and validates the quality of cold jet measurements acquired from both facilities. This is an encouraging comparison which validates the potential to develop noise reduction concepts in small scale jets that accurately simulate the acoustics issuing from the actual engine exhausts.

Closer inspection of the details of the small scale nozzles used in conducting the experiments at Penn State shows that the internal nozzle surface is slightly rougher at this scale level in comparison with the moderate scale nozzle. The lip thickness is also not exactly reproduced, due to the limitations of the rapid prototyping technique and the brittleness of the material used. Therefore, the thickness of the boundary layer at the exit plane of the nozzle may not scale perfectly with the moderate scale measurements. In spite of these differences, the comparisons are very good. This observation is in agreement with a previous study from Viswanathan [8] who validated the effect of the state of the flow at the nozzle exit plane. The conclusion from that study was that the radiated noise is insensitive to the state of the flow and the thickness of the boundary layer at the nozzle exit plane, provided the Reynolds number of the jet flow exceeds approximately 400,000 .

The current comparisons of unheated jet measurements were conducted in the geometric far field, with sound measurements being made well over 100 jet diameters from the nozzle. The term 'geometric far-field' is used when the noise is approximated by a point source causing the noise to radiate spherically so the sound intensity is reduced by $6 \mathrm{~dB}$ for per doubling of distance from the noise source. By maintaining the far field measurements in treating the noise as a compact point source emitting from the nozzle exit plane, the experimental results can be extrapolated to various non-dimensional distances for comparisons as is commonly done (see Ref. 9, 10, 11). This issue is of particular importance when working with supersonic jets, as will be covered later.

The data in Figs. 5 and 6 are representative of similar spectra recorded with numerous pressure ratio conditions. Such results show that the small scale jets approximately replicate the acoustics issuing from the moderate scale jets under over-expanded and under-expanded conditions. Moreover, the demonstration of the cross- 
scale comparison from the unheated jet experiments, measured in the far field, shows that the small scale unheated jets have been able to simulate the acoustic field generated by moderate scale unheated jets. This is an encouraging first step in making cross-scale comparisons with the heated jet measurements.

\section{B. Heat simulated jets and heated jets measurements}

The heat simulated jet experiments were conducted with helium-air mixture jets at Penn State, following the methodology established during previous studies $[2,3]$. Figure 7 shows acoustic spectra measured with the GE nozzles with $A R=1.295$ conducted with single stream jets at both Penn State and NASA GRC. The data recorded using the $M_{d}=1.65(A R=1.295) \mathrm{GE}$ nozzle operating at a pressure ratio of $N P R=3.0$ produces an average exit Mach number $M_{j}=1.36$, and the simulated temperature ratio $T T R=2.6$ for Penn State and TTR $=2.5$ for NASA. All acoustic levels have been adjusted to $R / D=100$ as in Figs. 5 and 6 .

As in the case of the cold jets, the acoustic data for the small scale experiments compare favorably with the NASA moderate scale data across the frequency range. The individual PSD components are always within $3 \mathrm{~dB}$ for both sets of data and most spectral components agree within $2 \mathrm{~dB}$.

The above demonstrations distinctly show that the small heat-simulated jet is capable of reproducing the acoustics issuing from a moderate size heated jet when the precise simulated properties are matched for the overexpanded CD nozzles, which are prevalent in practical supersonic aircraft. These data (and many other comparisons not shown here), show that the scaling of small heat simulated jets measurements are in reasonable agreement provided that comparisons are made in the far field past $R / D=100$, with identical nozzles, and with matching Mach number and total temperature ratio. In this context "reasonable agreement" can be quantified to state that agreement of individual portions of the spectra agrees within 2 to $3 \mathrm{~dB}$ and the overall sound pressure levels agree within $2 \mathrm{~dB}$.

\section{Small unheated bleed flow effect on the noise emission}

The most common configuration for the NASA HFJER is with co-annular dual flow with a very low bypass ratio $(B P R=0.3)$ of unheated air. Such a configuration consists of a core flow surrounded by a thin annulus of cold air, whose initial thickness is less than $10 \%$ of the core radius (shown schematically in Fig. 4). This configuration of nozzle is reasonably close to actual supersonic aircraft engines in which the annular fan flow plays a major role in cooling liner components of the engine. It does however introduce some uncertainty in how best to match these low $B P R$ exhaust jet acoustic experiments to single stream model jets for which a considerable amount of acoustic data are available. The experiments producing acoustic spectra are plotted in Fig. 8 as a head-to-head single flow versus dual flow acoustic data comparison.

First, the HFJER was operated both as single stream jet and in its standard configuration as a dual flow rig with a GE design nozzle with $M_{d}=1.5$. Figure 8 compares data from the same facility when the single stream TTR matched the core flow total temperature $\operatorname{TTR}_{\text {core }}(=3.2)$ and also when it matched to a hypothetical mixed flow temperature ratio $T T R_{\text {mix }}(=2.7)$ that assumes the core and cold annular flow to be fully mixed. As noted earlier, the bypass ratio was 0.3 in the dual flow experiment. In Fig. 8 the difference between the two single-stream jet noise curves is roughly $3 \mathrm{~dB}$ for frequencies below the peak, in line with expectations from increasing jet velocity with increased temperature. The temperature change also affects the shift in peak frequency in the data in the aft quadrant and there is some difference in the high frequency roll off such that the colder jet produces more high frequency noise at shallow aft angles, but less high frequency noise on the sideline. It is believed that the explanation for this is the rotation of the peak noise emission direction toward steeper angles from the jet (and further from the jet axis) for the hotter single stream jet. The dual stream noise spectra generally lies between the two single-jet curves at low frequencies and follows the higher temperature (core conditions) single jet at high frequencies. For this realistic CD nozzle geometry operating in an over-expanded condition matching the core conditions seems to produce the best match to the more realistic dual stream data.

Having quantified the error in simulating a low-bypass ratio practical CD nozzle with a single-stream jet, the next step is to add the effect of scale. The small-scale rig at Penn State was run in a similar fashion, with two total temperature values that match first the corresponding HFJER $T T R_{\text {core }}$ and second, the $T T R_{m i x}$ value. The results are plotted in Fig. 9. Note that this case uses a slightly different nozzle than Fig. 8, with a $M_{d}=1.65$; however, both cases are the faceted CD nozzles operating at the over-expanded condition. Here, the jet noise from the two singlestream jets at small scale differ by as much as $5 \mathrm{~dB}$ at low frequencies, more than did the same cases in the larger rig. Here the single stream (PSU jet), run at simulated temperatures matching the dual flow fully mixed temperature condition produced better agreement with the dual stream data at low frequencies. At higher frequencies and aft angles, the single-stream jet matching the core conditions of the dual stream jet best agreed with the dual stream jet, 
in the same way as the medium scale experiment did. It is noted that both the Penn State and the GRC data have shock screech components at virtually identical frequencies that are quite close in spectral level, assuring that the shock structure and details of the shock noise are well represented across the model scales (and with different methods of obtaining a "heated jet"). However, it can be concluded that there are imperfections in the matching of acoustic data between single stream and dual stream $(B P R=0.3)$ jets at frequencies below the peak frequency no matter what matching condition is used.

\section{Confirming experiments}

As an additional test of the validity of the Penn State data, comparisons have been conducted with a different data set, namely heated supersonic jet acoustic data measured by Seiner and Norum at the NASA Langley Research Center (LaRC) [12]. To do this requires that a more common nozzle geometry be used, a convergent-divergent nozzle with a contoured divergent section designed using the method of characteristics to be shock-free at its exit at $M_{j}=1.5$. Making this change also removes shock-associated noise from the comparison. In this case the LaRC experiments were conducted with a $4.27 \mathrm{~cm}$ (1.68 in) exit diameter ideally contoured axisymmetric CD nozzle operating ideally expanded at a $N P R=3.6$ and $T T R=2.25$. The Penn State experiments used a $12.5 \mathrm{~mm}(0.5 \mathrm{in})$ exit diameter contoured axisymmetric $\mathrm{CD}$ nozzle operating at a $N P R=3.6$ and $T T R=2.2$. The spectral comparisons are shown in Fig. 10, with the data agreeing across the spectra for $90^{\circ}$ and $100^{\circ}$ to within $+/-1.5 \mathrm{~dB}$. The biggest discrepancy in the data, of $4 \mathrm{~dB}$ at the peak frequency and $60^{\circ}$ polar angle, can be partially accounted for by the differences in the $R / D$ location of the two data sets. As noted earlier, when the distance to the microphones is different between two experiments, differences in spectral level are often most apparent at polar angles around 50 to $60^{\circ}$ where the gradient in OASPL with polar angle is the greatest. At the most aft angle shown $\left(40^{\circ}\right)$, the PSU data are less than $3 \mathrm{~dB}$ below the LaRC data.

Continuing with the approach of comparing acoustic data acquired at different facilities, Fig. 11 presents a comparison of the LaRC single jet data shown in Fig. 10 with those of GRC HFJER run in both single-flow (TTR = 2.25 ) and dual-flow $\left(T T R_{\operatorname{mix}}=2.25\right)$ modes using a contoured axisymmetric CD nozzle with $M_{d}=1.5$. The first observation is that for this ideally expanded flow, operation of the GRC HFJER facility in the dual flow mode produces spectra that more closely match the smaller LaRC facility jet spectra than do the spectra in the single jet mode. All three jet data sets agree well at the broadside angles of $100^{\circ}$ and $90^{\circ}$. By $60^{\circ}$ there appears a systematic discrepancy in levels at frequencies below the peak, where the smaller rig produces more noise than the larger rig. At angles aft of $60^{\circ}$ the GRC HFJER data are lower than the LaRC data by increasing amounts, being $5 \mathrm{~dB}$ different at $40^{\circ}$ to the jet axis. At this point it is noted that the feature of higher noise levels at low frequencies for the smaller rigs is consistently present in the data of Figs. 7,9 and 11. This feature will be addressed further later in this section of the text.

One other major feature has been present in these datasets that merits attention: the higher frequencies at which the HFJER data show a very strong rise (an upward "hook"). This sharp rise is not visible in raw data, but results from performing the atmospheric attenuation correction to obtain "lossless" data. Such a rise is slightly apparent in both the Penn State and the LaRC data, both of which are not resolved to the same high non-dimensional frequency as is the GRC data. This feature is most significant at the angles of strongest noise emission, e.g., aft angles, and typically appears at supersonic, hot jet conditions, as discussed in Ref. [13]. The physical explanation seems to be the presence of strong nonlinear distortion that is present in very high amplitude noise that is undergoing wave steepening (as discussed in several references, for example Ref. [13]). It appears from the data presented here, and much more which was not presented, that the increase in sound amplitude in absolute terms going from the $\sim 50$ $\mathrm{mm}$ (2") nozzles to $\sim 100 \mathrm{~mm}$ (4") nozzles is sufficient to cause the onset of nonlinear propagation within the same relative measurement distance.

It is noted that the rising spectral "hook" mentioned in the preceding paragraph is not seen in the Penn State data of Figs. 7 and 9. In both those data sets the small scale data agree with the moderate scale NASA data quite well but do not extend in frequency resolution to the point where the lossless spectral content begins to rise. The microphones response limited the resolution. An experiment was conducted at Penn State however, in which the microphones were placed 280 jet diameters from the nozzle exit over the range of polar angles. The data, presented in Fig. 12, show an almost identical shape as the moderate scale data, just shifted to a lower frequency. Such a shift in the location to the onset of the nonlinear distortion is consistent with the discussion presented by Petitjean et al. [13] on the onset of such effects. Additionally Saxena et al. [14] and Lee et al. [15] have developed a nonlinear propagation computational model that predicts the general high frequency lift-up feature that is seen in the data of Fig. 12. In summary, nonlinear propagation effects require the initially very high sound pressure levels, long 
propagation ranges within the facility, resolution of the frequency spectra to high frequencies and finally, properly applied correction of the spectra to remove the effect of atmospheric attenuation (to produce lossless data).

To summarize the results of the comparisons made with the data presented in Figs. 7 to 10 it is apparent that there is consistency between the single stream Penn State and LaRC data operating at similar jet mean flow (including $T T R$ ) conditions with an ideally expanded, shock-free jet flow. For realistic nozzle models running overexpanded conditions, the Penn State and GRC data match well when both are operated in single-stream mode with the following exceptions. For ideally expanded nozzles, the larger GRC nozzle exhibited a significantly lower noise in the low frequency range (below the peak frequency) at aft angles, than the smaller single-flow rigs. In using single-flow rigs to simulate low bypass ratio jet flows there is a choice to be made whether to match the core conditions or the fully mixed conditions. For the over-expanded realistic nozzle flows, there is a significant impact of the bypass flow. For the ideally expanded shock-free nozzle at $M=1.5$ there was little impact of the cold bypass stream at this bypass ratio of 0.3 . However, the larger rig produced data that were slightly but measurably lower than the smaller rigs for this nozzle. At present these contradictions are unresolved.

At this point it is appropriate to mention the possibility that the annular co-flow that surrounds the core jet could serve to eliminate or reduce the Mach wave radiation as explained by Papamoschou [16]. If the parameters of the annular flow were assumed to be retained following convection through the nozzle, the convection velocity of the turbulence on the outer shear layer would be reduced to below the ambient acoustic velocity thus eliminating Mach wave radiation. It seems rather unlikely that such could be the case in precisely the manner suggested by Papamoschou. It is anticipated that the annular co-flow, which starts upstream of the nozzle throat, would become mostly mixed with the core flow, at least in the outer portion of the jet at the exit of the jet. Thus the criteria used by Papamoschou to predict the parameters for Mach wave elimination are likely not to be satisfied. The outer shear layer however, will certainly be affected, resulting in reduced levels of mean shear which would be expected to slightly reduce the radiated noise, a result that is consistent with observed data.

One focus of future experiments will be to examine the effect of the thin layer of cold air surrounding the hot jet in the dual flow experiments. Recognizing that there remains an unresolved contradiction in the best way to match TTR operating conditions when comparing the acoustic data of the dual flow facility with those from the single flow facility, one notices a previously unmentioned issue. The hot jets that compared well using the $T T R_{\text {core }}$ were all conducted at a Reynolds number of 2 million or greater. The jets that compared well with the HFJER $T T R_{\text {mix }}$ were all at $R_{e}<900,000$. Although it is premature to draw a definitive conclusion, the lower Reynolds number jet data have a very similar additional noise component that was identified by Viswanathan [17] with heated subsonic jets at $M=0.7$. This issue is being addressed with additional experiments and data processing.

\section{E. Near field / Far field measurement issues}

During the process of comparing acoustic data acquired in experiments with different scale nozzles it is quite easy to get sidetracked examining data in which not every physical parameter was maintained constant that needed to be. One such example is the microphone radial location during experiments with nozzles of two scales. There is considerable experience with cold jets to conclude that if the microphone(s) are positioned 50 to 70 radii away from a jet (exit plane) then good accuracy can be obtained using the $6 \mathrm{~dB}$ per doubling of distance to scale acoustic amplitude levels to a common radius. Such is not the case with hot supersonic jets. Two examples of this effect in the acoustic spectra are shown in Figs. 13 and 14.

Figure 13 shows spectra of acoustic measurements performed (at Penn State) in the acoustic field of two supersonic jets exiting from CD nozzles operating at $M_{j}=1.5(N P R=3.6)$ and simulated total temperature ratios of $T T R=3.2$. The microphones for both jets were positioned at a radius of $1.78 \mathrm{~m}(70 \mathrm{in})$ on a polar arc originating at the nozzle exit. Since the two nozzles used in these measurements were 12.7 and $25.4 \mathrm{~mm}$ in diameter, the $R / D$ value for the experiments were $R / D=140$ and 70 respectively. In the data of Fig. 13, both sets of data were then scaled (by spherical spreading) to $R / D=100$. Note that the spectral data are in reasonable agreement but differences of about $3 \mathrm{~dB}$ in magnitude are apparent between data of two size nozzles (and measurement $R / D$ positions). The discrepancies are most apparent at frequencies below the peak amplitude level and in the polar angle range from $40^{\circ}$ to $60^{\circ}$ from the jet axis corresponding to the afore mentioned region of largest gradient in the OASPL with polar angle.

Figure 14 shows very similar data as in Fig. 13 this time recorded with a heated jet exhausting from the NASA GRC HFJER facility fitted with a CD nozzle and $30 \%$ bypass unheated air. In this case the acoustic data were recorded simultaneously with microphones positioned on an arc at $R / D=147$ and on a linear array displaced parallel from the jet axis by $33 D$. The latter data, noted on the figure as at an average radius of $R / D \sim 50$, are clearly not in the geometric far field. The plotted data are scaled to a non-dimensional radial position of $R / D=100$ and 
discrepancies in the spectral level are observed as with the Penn State small scale jet measurements of the previous figure.

Such discrepancies between the measurements made at the threshold of the near to far field and those that are clearly in the geometric far field are significant and render the comparisons of the effect of other parameters, in some cases, less effective. In essence the problem is much more acute with hot and heat-simulated jets, than it is with cold supersonic jets. As demonstrated by Lee and Bridges [18] and McLaughlin et al. [19] the dominant region of noise sources in hot supersonic jets center around a non-dimensional axial distance $x / D=13$, whereas the location for comparable cold jets is around $x / D=8$. This shift of the dominant region of noise sources as well as the extended length of the source region is enough to distort the spherical spreading scaling of acoustic data to different radial positions when the measured microphones on a polar arc originate at the nozzle exit. This reason makes it more important to perform comparison measurements at the same non-dimensional radial positions. Such is not always possible when making comparisons to data recorded in experiments performed in past years.

\section{Conclusions}

Careful measurements and normalization were employed to produce direct comparisons between small scale jet acoustic measurements made at Penn State and moderate scale measurements from NASA GRC. In most cases the supersonic nozzles were of identical geometries (to engineering accuracy) and closely replicated the exhaust nozzles of GE 404 class turbojet engines. Other experiments were performed with contoured converging-diverging (CD) nozzles producing minimal shock cells in the flow field when operated at perfectly expanded pressure ratios. Initial experiments were performed with unheated, pure air jet flows. For these cold jets, non-dimensional acoustic data measured at identical scaled radial distances were shown to agree within $+/-1.5 \mathrm{~dB}$ across the spectra at numerous polar angles. The Reynolds numbers for all cold jet data presented exceeded 900,000 and imperfections in nozzle surface roughness and lip thickness did not appear to have any significant effect.

For hot jet comparisons, the small scale (Penn State) experiments simulated the hot-jet velocity conditions of experiments conducted at NASA GRC and NASA LaRC by using helium-air gas mixtures. Comparisons of the acoustic fields (measured in small and moderate sized facilities) of hot jets from practical CD nozzles in typical over-expanded conditions produced very good agreement on $O A S P L$ and spectra, typically within $+/-1.5 \mathrm{~dB}$. Comparisons for ideally expanded nozzles operating at their design Mach numbers showed good agreement between rigs of similar scale (nozzle diameters less than $50 \mathrm{~mm}(2 \mathrm{in})$ ), but not as good agreement between the small and medium scale rigs, particularly at frequencies below the peak amplitude values.

Realistic exhaust systems often have unmixed bypass flows exiting the nozzle, a feature incorporated into the medium scale rig. For over-expanded jet conditions in realistic CD nozzles, there was an impact of the bypass flow on the noise; the impact on ideally expanded flows from shock-free nozzles was much smaller. Comparisons of the small Penn State rig data with hot jet supersonic jet data from the NASA HFJER operating with low bypass ratio $(B P R=0.3)$ showed reasonable agreement $(+/-1.5 \mathrm{~dB})$ provided the single stream total temperature ratio TTR was matched to the theoretical mixed flow $T T R_{\text {mix }}$ of the HFJER jet. Comparison of measurements made in the HFJER facility operating in single-flow and dual-flow $(B P R=0.3)$ modes demonstrated that more accurate data were obtained when the single stream $T T R$ was matched to the $T T R_{\text {core }}$ of the dual stream jet. This discrepancy in the acoustic data of the two kinds of jets is being investigated further with additional experiments and examination the effect of the annular bypass stream and of a possible Reynolds number effect.

The unresolved issue of nonlinear propagation, which is just now being recognized in supersonic jet data, was demonstrated in rigs of both small and medium scale, particularly at polar angles of maximum acoustic output. The unresolved part is how to properly scale and correct for this effect, as demonstrated in the data presented here, without a major computational effort as reported by Saxena et al. [14]. This becomes more of a problem as measurement locations are moved further away from the jet to be truly in the geometric far field.

Finally, data are presented that examine the accuracy of scaling measurements that are made in the outer regions of the near field to far field distances. These data demonstrate that measurements in supersonic jets must be made much further away from the jet to be in the geometric far-field. Presumably this is caused by the position of the dominant noise sources moving significantly downstream of the nozzle exit along with an increase in the lengthwise extent of the dominant source region.

\section{Acknowledgments}

This work was performed under funding from the Strategic Environmental Research and Development Program, Project Number WP-1583. The Penn State authors take pleasure in acknowledging the direct assistance of Dr. Jeremy Veltin with the experiments and the data interpretation, and helpful discussions with Prof. Philip Morris of 
Penn State and Dr. Steven Martens of GE. Dr. Brenda Henderson of NASA also made significant contributions to the manuscript during final preparation. The NASA experiments were conducted under the Fundamental Aeronautics Program, Supersonics Project. Model hardware was loaned to NASA by the General Electric Company.

\section{References}

[1] Viswanathan, K., "Does a model scale nozzle emit the same jet noise as a jet engine," AIAA Journal, Vol. 46, No. 2, 2008, pp. 336-355.

[2] Doty, M. J. and McLaughlin, D. K., "Acoustic and mean flow measurements of high-speed, helium-air mixture jets," International Journal of Aeroacoustics, Vol. 2, No. 2, 2003, pp. 293-334.

[3] Papamoschou, D., "Acoustic simulation of coaxial hot air jets using cold helium-air mixture jets," Journal of Propulsion and Power, Vol. 23, No. 2, 2007, pp. 375-181.

[4] Bridges, J. and Brown, C. A., "Validation of the small hot jet acoustic rig for aeroacoustic research," AIAA Paper No. 2005-2846, 2005.

[5] Bridges, J. and Brown, C. A., "Parametric testing of chevrons on single flow hot jets," AIAA Paper No. 20042824, 2004.

[6] Kuo, C.-W., Veltin, J., and McLaughlin, D. K., "Methods to improve the accuracy of acoustic measurements in small scale high speed jets," AIAA Paper No. 2009-3251, 2009.

[7] Kuo, C.-W., Veltin, J., and McLaughlin, D. K., "Acoustic measurements of models of military style supersonic nozzle jets," AIAA Paper No. 2009-18, 2009.

[8] Viswanathan, K. and Clark, L. T., "Effect of nozzle internal contour on jet aeroacoustics," International Journal of Aeroacoustics, Vol. 3, No. 2, 2004, pp. 103-135.

[9] Ahuja, K. K., "Designing clean jet-noise facilities and making accurate jet-noise measurements," International Journal of Aeroacoustics, Vol. 2, No. 3\&4, 2003, pp. 371-412.

[10] Viswanathan, K., "Instrumentation considerations for accurate jet noise measurements," AIAA Journal, Vol. 44, No. 6, 2006, pp. 1137-1149.

[11] Koch, L. D., Bridges, J., Brown, C., and Khavaran, A., "Experimental and analytical determination of the geometric far field for round jets," Noise Control Engineering Journal, Vol. 53, No. 1, 2005, pp.20-28.

[12] Seiner, J. M. and Norum, T. D., Jet noise data provided by private communication, 2001.

[13] Petitjean, B. P., Morris, P. J., and McLaughlin, D. K., "On the nonlinear propagation of shock-associated jet noise," AIAA Paper No. 2005-2930, 2005.

[14] Saxena, S., Morris, P. J., and Viswanathan, K., "Algorithm for the nonlinear propagation of broadband jet noise," AIAA Journal, Vol.47, No. 1, 2009, pp. 186-194.

[15] Lee, S., Morris, P. J., and Brentner, K.S., "Nonlinear Acoustic Propagation Predictions with Applications to Aircraft and Helicopter Noise," AIAA Paper No. 2010-1384, 2010.

[16] Papamoschou, D., "Mach wave elimination in supersonic jets," AIAA Journal, Vol. 35, No. 10, 1997, pp. 16041611.

[17] Viswanathan, K., “Aeroacoustics of hot jets," J. Fluid Mech., Vol. 516, 2004, pp. 39-82. 
[18] Lee, S. S. and Bridges, J., "Phased-array measurements of single flow hot jets," AIAA Paper No. 2005-2842, 2005 .

[19] McLaughlin, D. K., Kuo, C.-W., and Papamoschou, D., "Experiments on the effect of ground reflections on supersonic jet noise," AIAA Paper No. 2008-22, 2008.

Table 1 Operating conditions of measurements in each figure.

\begin{tabular}{|c|c|c|c|c|c|c|c|c|}
\hline & Curve ID & $\begin{array}{c}\text { Design \& Jet } \\
\text { Mach No. } \\
M_{d} \& M_{j}\end{array}$ & $\begin{array}{c}\text { Total Temp. } \\
\text { Ratio, TTR } \\
\left.T_{T R_{\text {core }}\left(T T R_{\text {mix }}\right)}\right)\end{array}$ & $\begin{array}{c}\text { Acoustic } \\
\text { Mach No. } M_{a}\end{array}$ & $\begin{array}{c}\text { Nozzle Exit } \\
\text { Diameter } \\
D(\mathrm{~cm})\end{array}$ & $\begin{array}{c}\text { Measured } \\
\text { Distance } \\
R / D\end{array}$ & $\begin{array}{c}\text { Char. Freq. } \\
f_{c}\left(U_{j} / D_{j}\right) \\
\mathrm{Hz}\end{array}$ & $\begin{array}{c}R_{e} \\
\text { based on } \\
T T R_{\text {mix }}\end{array}$ \\
\hline \multirow{2}{*}{ Fig. 5} & PSU & \multirow{2}{*}{$1.65 \& 1.56$} & \multirow{2}{*}{1.0} & \multirow{2}{*}{1.28} & 1.8 & 99 & 25,315 & 992,200 \\
\hline & HFJER & & & & 12.9 & 116 & 3,351 & $7,356,200$ \\
\hline \multirow{2}{*}{ Fig. 6} & PSU & \multirow{2}{*}{$1.5 \& 1.56$} & \multirow{2}{*}{1.0} & \multirow{2}{*}{1.28} & 1.7 & 104 & 25,321 & 972,900 \\
\hline & HFJER & & & & 12.3 & 121 & 3,469 & $6,965,500$ \\
\hline \multirow{2}{*}{ Fig. 7} & PSU & \multirow{2}{*}{$1.65 \& 1.36$} & 2.6 & 1.86 & 1.7 & 104 & 38,031 & 499,200 \\
\hline & HFJER & & 2.5 & 1.84 & 12.3 & 121 & 6,200 & $1,600,000$ \\
\hline \multirow{3}{*}{ Fig. 8} & \multirow{3}{*}{ HFJER } & \multirow{3}{*}{$1.5 \& 1.36$} & 3.2 & 2.08 & \multirow{3}{*}{12.3} & \multirow{3}{*}{121} & 6,326 & $1,219,600$ \\
\hline & & & 2.5 & 1.88 & & & 5,706 & $1,552,900$ \\
\hline & & & $3.2(2.7)$ & $2.08(1.85)$ & & & 5,384 & $1,442,700$ \\
\hline \multirow{3}{*}{ Fig. 9} & \multirow{2}{*}{ PSU } & \multirow{3}{*}{$1.65 \& 1.36$} & 3.0 & 2.03 & \multirow{2}{*}{1.8} & \multirow{2}{*}{99} & 41,694 & 460,300 \\
\hline & & & 2.6 & 1.86 & & & 38,031 & 499,200 \\
\hline & HFJER & & $3.0(2.6)$ & $2.03(1.86)$ & 12.9 & 116 & 5,334 & $1,675,500$ \\
\hline \multirow{2}{*}{ Fig. 10} & PSU & \multirow{2}{*}{$1.5 \& 1.5$} & 2.2 & \multirow{2}{*}{1.85} & 1.27 & 140 & 50,028 & 485,500 \\
\hline & LaRC & & 2.25 & & 4.27 & $\sim 100$ & 14,976 & 844,700 \\
\hline \multirow{3}{*}{ Fig. 11} & HFJER & \multirow{3}{*}{$1.5 \& 1.5$} & 2.2 & 1.85 & \multirow{2}{*}{10.2} & \multirow{2}{*}{147} & \multirow{2}{*}{6,269} & \multirow{2}{*}{$2,084,500$} \\
\hline & НTЕК & & $2.6(2.2)$ & $2(1.85)$ & & & & \\
\hline & LaRC & & 2.25 & 1.85 & 4.27 & $\sim 100$ & 14,976 & 844,700 \\
\hline \multirow{2}{*}{ Fig. 12} & PSU & \multirow{2}{*}{$1.5 \& 1.5$} & \multirow{2}{*}{2.2} & \multirow{2}{*}{1.85} & 1.27 & 280 & 50,028 & 485,500 \\
\hline & HFJER & & & & 10.2 & 147 & 6,269 & $2,084,500$ \\
\hline Fio 13 & PSU & 15815 & 32 & 223 & 1.3 & 140 & 58,914 & 431,200 \\
\hline Fig. 13 & PSU & $1.5 \propto 1.5$ & 3.2 & 2.25 & 2.5 & 50 & 29,390 & 865,100 \\
\hline $\mathrm{Fi}$ & HEIFP & 158 & $26(27$ & $2(185)$ & 10 & 147 & 6260 & 2084500 \\
\hline Fig. 14 & HFJEK & $1.5 \propto 1.5$ & $2.0(2.2)$ & $2(1.80)$ & 10.2 & $\sim 50$ & 0,209 & $2,084,500$ \\
\hline
\end{tabular}

All measurements presented are extrapolated to the scaled propagation distance $(R / D)$ equal to 100 . 


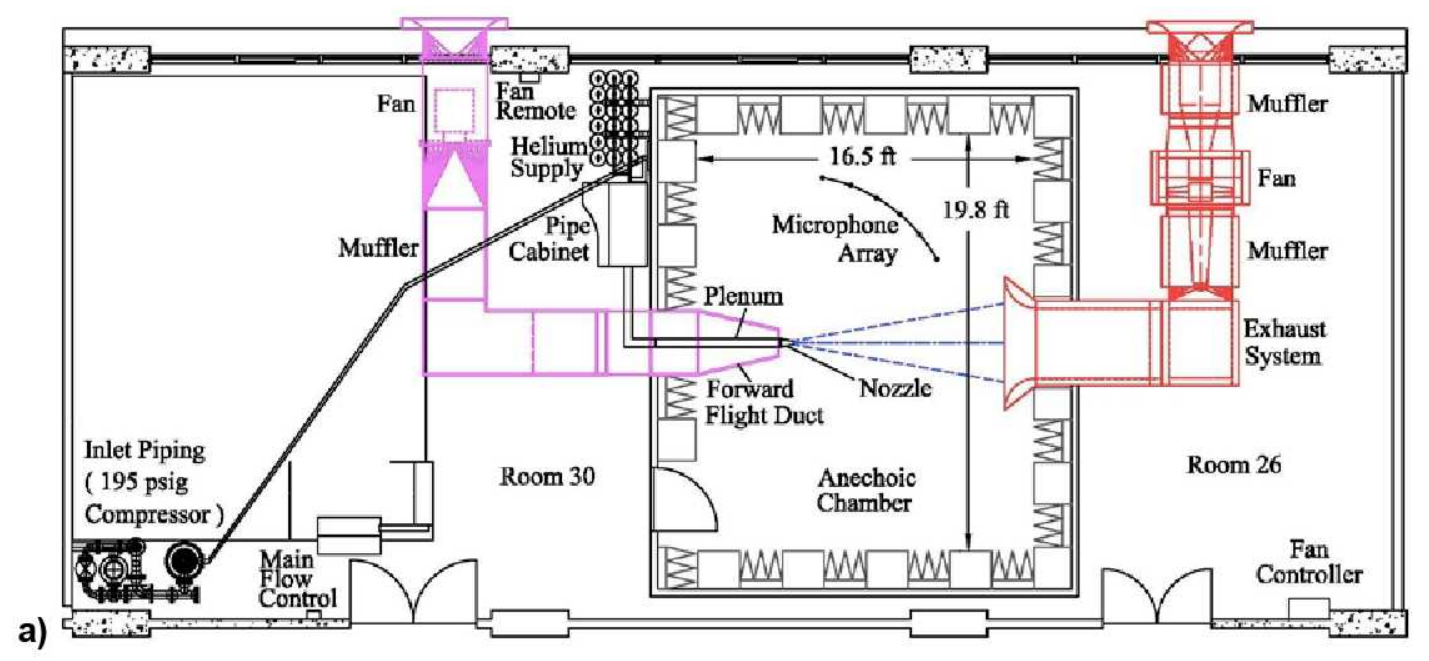

a)

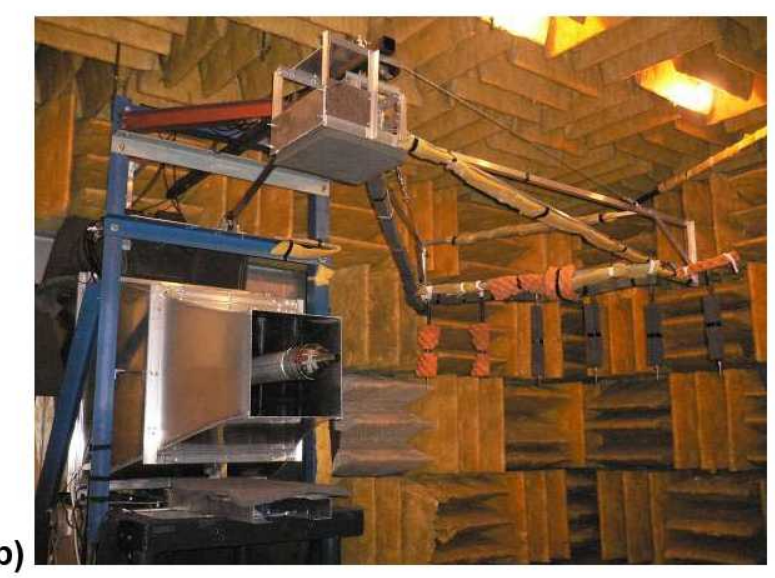

Fig. 1 The Pennsylvania State University high speed jet noise facility a) schematic b) photograph.

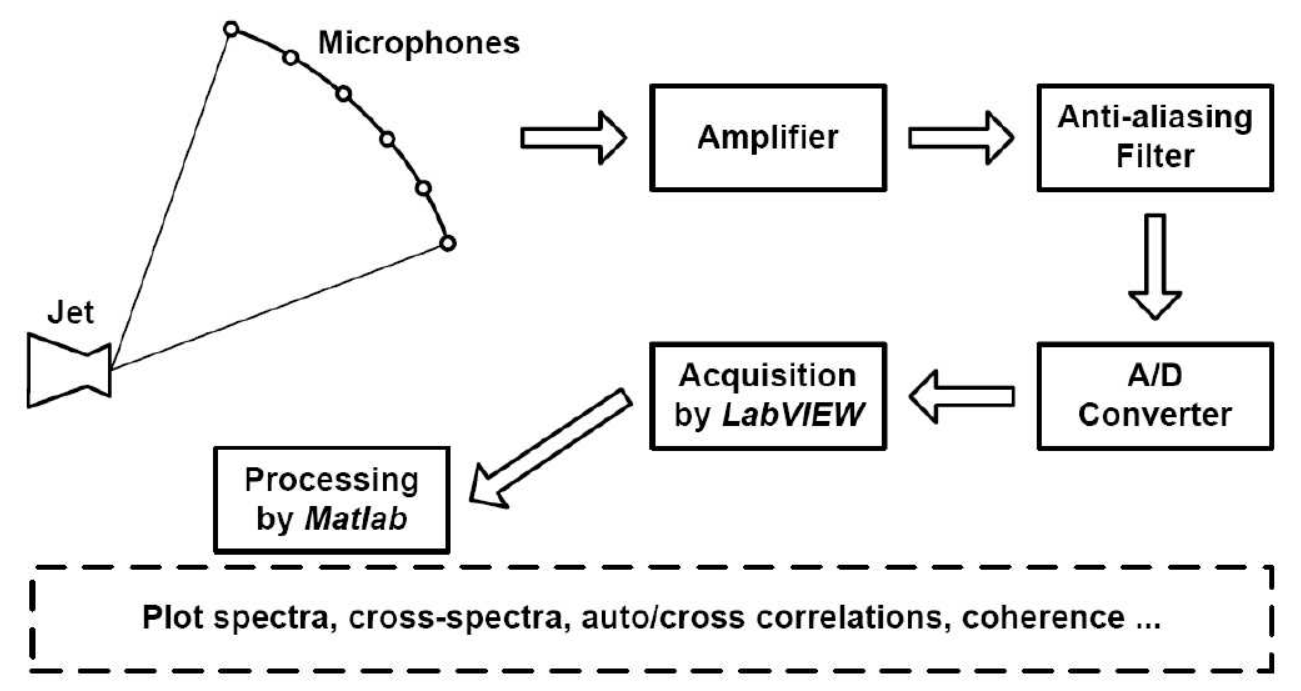

Fig. 2 Flow chart of the data acquisition process. 


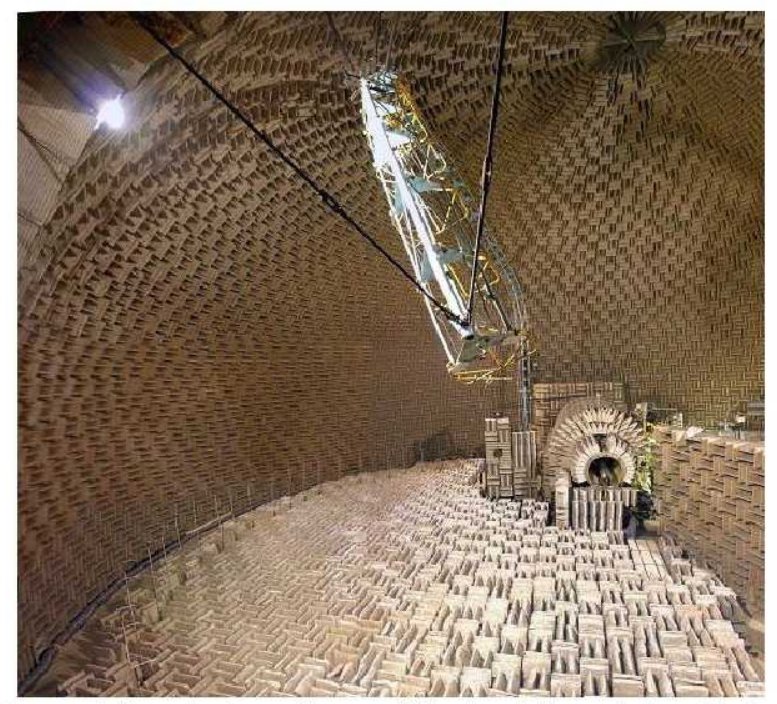

Fig. 3 The AeroAcoustic Propulsion Laboratory with dual flow jet exit rig in the NASA Glenn Research Center.

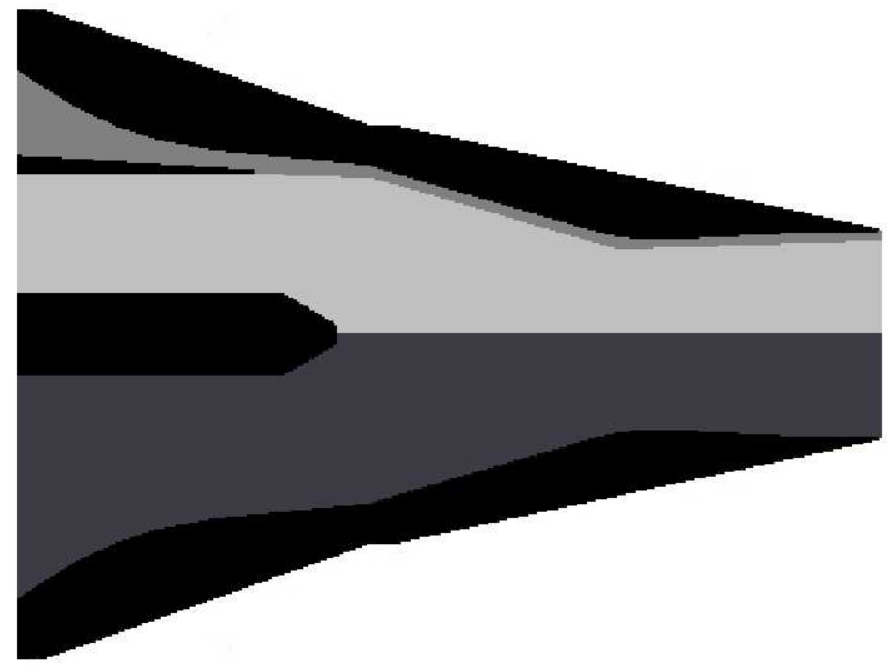

Dual stream with heated core jets and cold annual jets operated at the same nozzle pressure ratio

Single stream premixed with heated core jets and cold annual jets operated at the same nozzle pressure ratio

Fig. 4 Schematic of dual flow jet exit rig in the NASA Glenn Research Center with the bypass air for low bypass nozzle systems. 

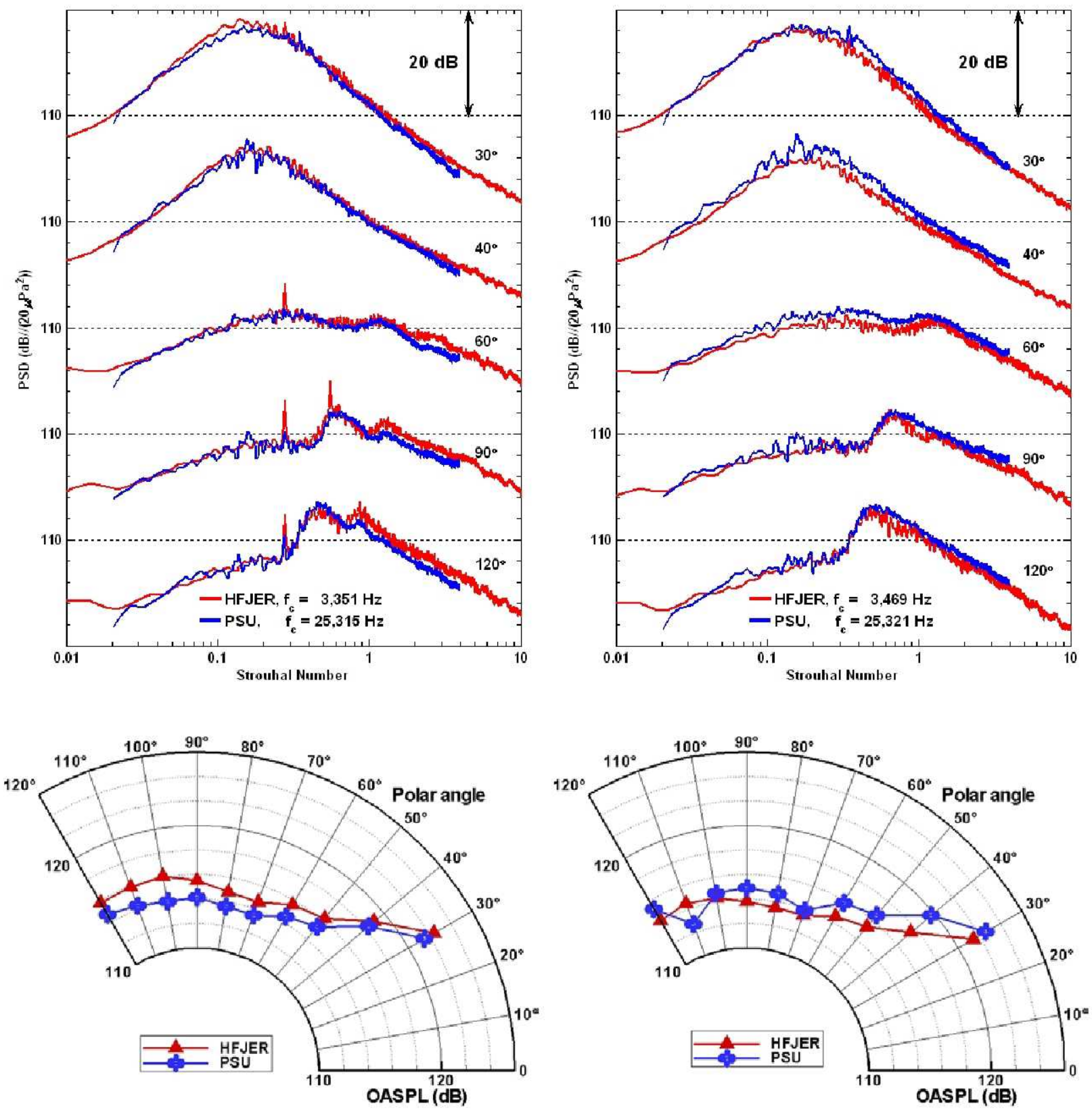

Fig. 5 Spectra and OASPL comparison of unheated jets from PSU and HFJER issuing from GE nozzle with $M_{d}=1.65$, $M_{j}=1.56$, and scaled to $R / D=100$.

Fig. 6 Spectra and OASPL comparison of unheated jets from PSU and HFJER issuing from GE nozzle with $M_{d}=1.5$, $M_{j}=1.56$, and scaled to $R / D=100$. 

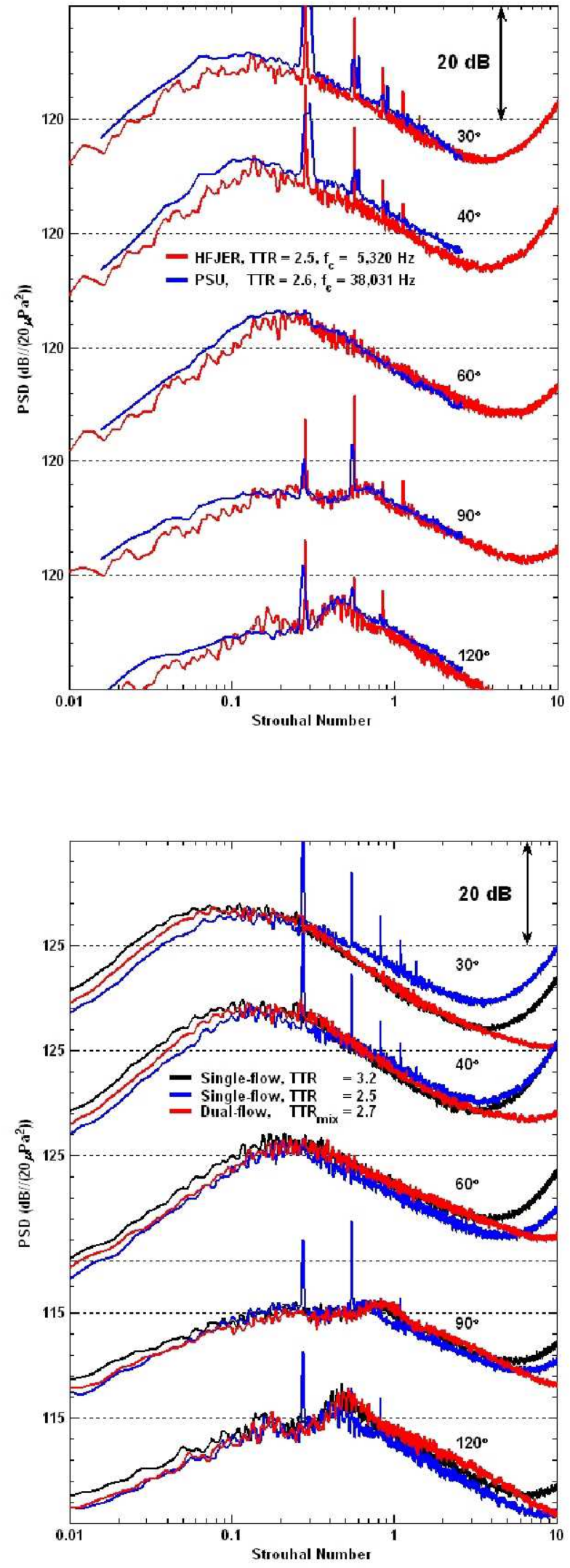

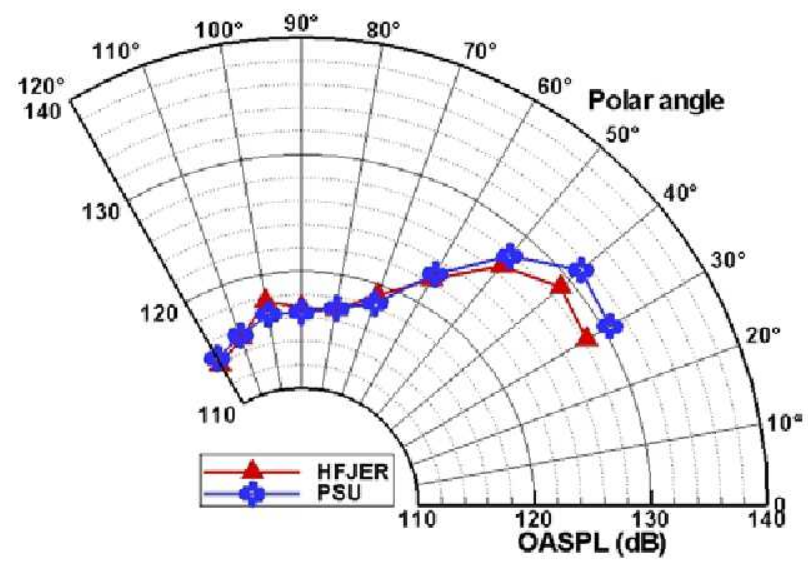

Fig. 7 Spectra and OASPL comparison of single stream jet with heat simulation (TTR = 2.6) from PSU and heated jet (TTR = 2.5) from HFJER both issuing from GE nozzle with $M_{d}=1.65$, $M_{j}=1.36$, and scaled to $R / D=100$.
Fig. 8 Spectra comparison among single-flow heated jets (TTR $=3.2$ and 2.5$)$ and dual-flow heated jet $\left(T T R_{\text {core }}=3.2\right.$, $T T R_{\text {mix }}=2.7$ with $\left.S t_{\text {dual }}=f D_{j} / U_{j \text { mix }}\right)$ with $B P R=0.3$ all from HFJER issuing from GE nozzle with $M_{d}=1.5, M_{j}=1.36$, and scaled to $R / D=100$. 


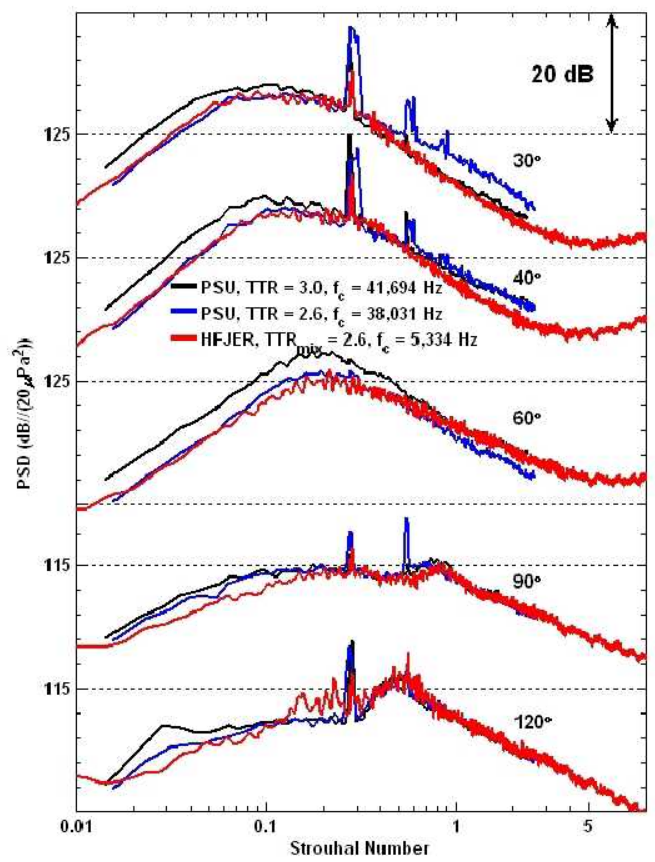

Fig. 9 Spectra comparison among heat simulated jets $(T T R=3.0$ and 2.6$)$ from PSU and heated jet $\left(T T R_{\text {core }}=3.0, T T R_{\text {mix }}=2.6\right)$ from HFJER all issuing from GE nozzle with $M_{d}=1.65$, $M_{j}=1.36$, and scaled to $R / D=100$.

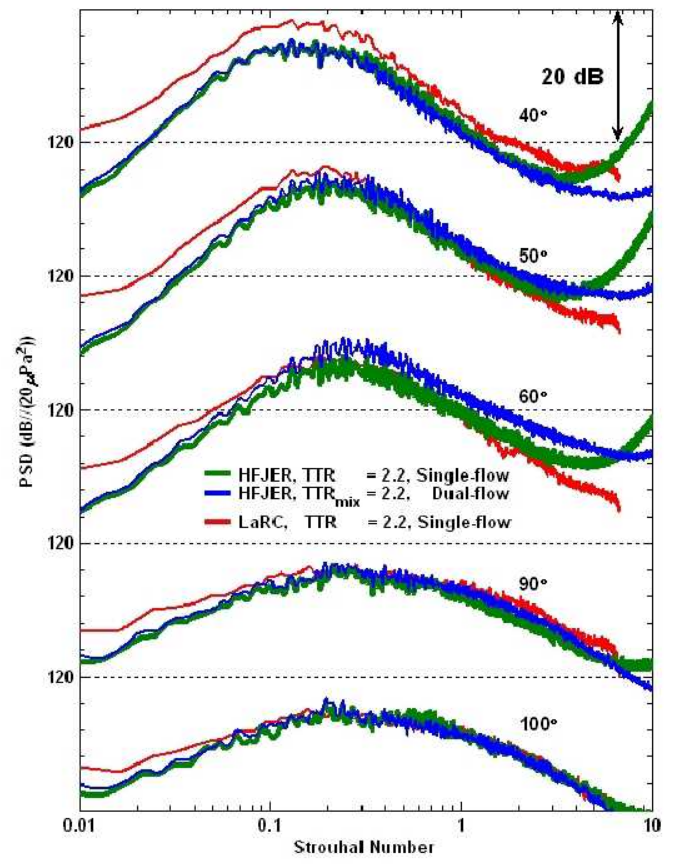

Fig. 11 Spectra comparison of heated jets with HFJER in single-flow $(T T R=2.2)$ and dual-flow $\left(T T R_{\text {mix }}=2.2\right)$, and LaRC in single-flow jet $(T T R=2.2)$, all issuing from CD nozzle with $M_{d}=1.5, M_{j}=1.5$, and scaled to $R / D=100$.

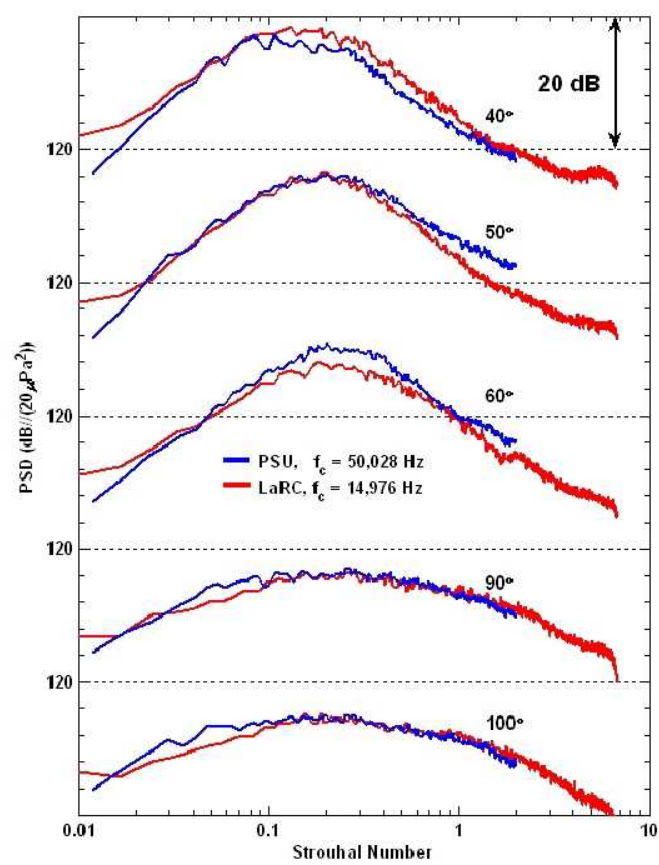

Fig. 10 Spectra comparison between heat simulated jet $(T T R=2.2)$ from PSU and heated jet $(T T R=2.25)$ from NASA Langley Research Center (LaRC) both issuing from CD nozzle with $M_{d}=1.5, M_{j}=1.5$, and $R / D=100$.

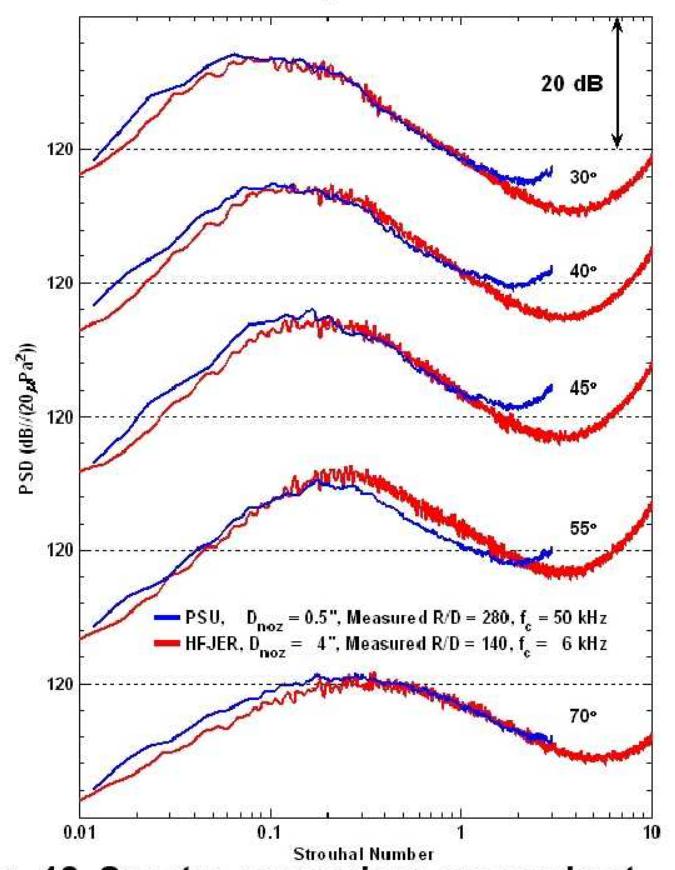

Fig. 12 Spectra comparison among heat simulated jet $(T T R=2.2)$ from PSU measured at $R / D=\mathbf{2 8 0}$ and heated jet (TTR = 2.2) from HFJER in single-flow measured at $R / D=140$ both issuing from CD nozzle with $M_{d}=1.5, M_{j}=1.5$, and scaled $R / D=100$. 


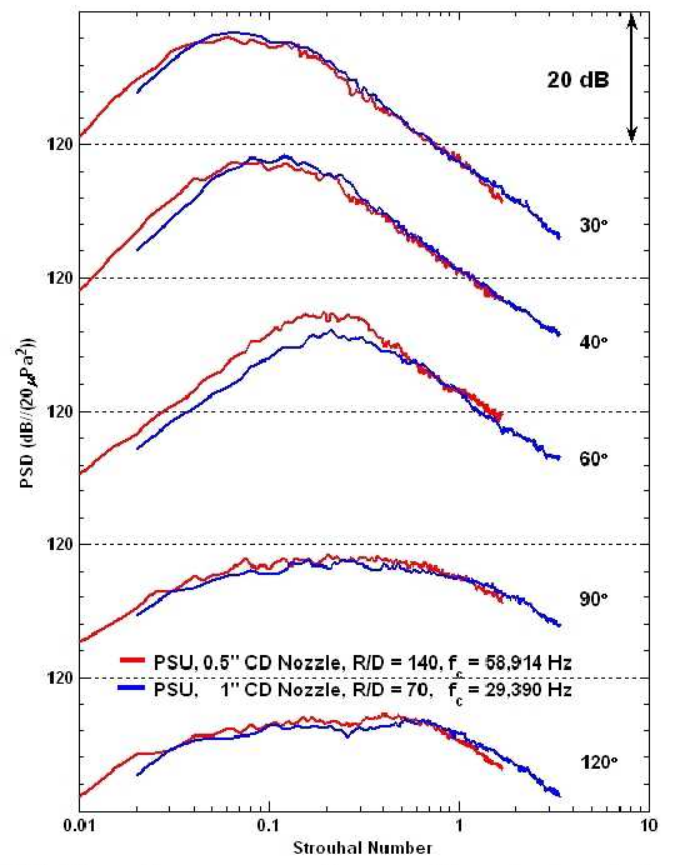

Fig. 13 Spectra comparison of heat simulated jets $\left(M_{j}=1.5, T T R=3.2\right)$ from PSU issuing from $C D$ nozzles with $M_{d}=1.5$, $D=0.5 "(1.3 \mathrm{~cm})$ and $1 "(2.5 \mathrm{~cm})$ respectively; scaled to $R / D=100$.

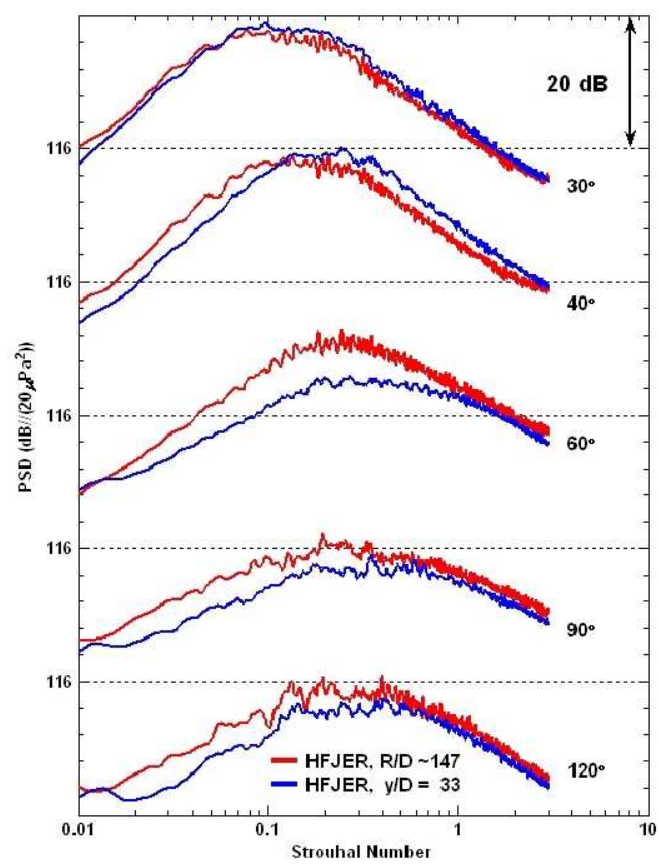

Fig. 14 Spectral comparison of experiments measured at various locations from HFJER issuing from CD nozzle with $M_{d}=1.5, M_{j}=1.5, T T R_{\text {core }}=2.6$, $T T R_{\text {mix }}=2.2$; scaled to $R / D=100$. 